\title{
Membrane Factor Method for large deflection response of beams and plates to intense dynamic loading
}

\author{
F. L. $\mathrm{Chen}^{1} \&$ T. X. Yu ${ }^{2}$ \\ ${ }^{1}$ Institute of Applied Physics and Computational Mathematics, China \\ ${ }^{2}$ Department of Mechanical and Aerospace Engineering, \\ Hong Kong University of Science and Technology, Hong Kong, China
}

\begin{abstract}
This paper deals with the large deflection of rigid-plastic structural members subjected to intense dynamic loads, such as explosive pressure and impact loadings. From the viewpoint of energy equilibrium, a theoretical procedure called the Membrane Factor Method was proposed by the authors in analyzing the dynamic plastic response of beams and plates with deflections in the range where both bending moments and membrane forces are of significance. The Membrane Factor Method is generalized and elaborated and its applications to various cases of dynamically loaded beams and plates are surveyed, with the expressions of the modifying membrane factors in each case being summarized and discussed. It is seen from comparison that the predictions by this method on the final deflections of beams and plates agree well with the corresponding experimental results for deflections up to 5-10 times of thickness. This procedure greatly improves the estimates from the bending-only theory and provides feasible complete response analyses, whose predictions are much more reliable than modal approximations.
\end{abstract}

Keywords: Membrane Factor Method, large deflection, plastic response, beam, plate, intense dynamic load.

\section{Introduction}

Large dynamic plastic deformation of structures is of significant interest in many engineering problems, such as safety calculations, hazard assessments, forensic 
investigations of ductile structures which are subjected to explosions or large dynamic loads producing an inelastic response.

Most of the earlier theoretical studies on dynamic plastic response of structures were limited to small deformation [1]. Nevertheless, the contradiction between the assumption on small deflection and the rigid-plastic idealization, which implies that the deflection caused by intensive loading is far beyond the elastic regime, greatly limits the practical validity of the small deflection solutions. Beams and plates under impact loading have been examined by many researchers theoretically or experimentally [2-8]. Experimental results revealed that membrane force play a remarkable role and even dominate the behaviour of structures when transverse deflection reaches or excesses the thickness [6-8]. But the complexity arising from both physical (plasticity) and geometrical nonlinearity makes large deflection problems rather complicated to treat theoretically when conventional methods are used, especially if dynamic effect is involved.

Large deflection, in general, may cause two kinds of effect on the dynamic plastic behaviour of structures: i) change in geometric configuration; and ii) development of the membrane, or in-plane, force. For a beam with end supports that prevent axial movement or a plate in most edge conditions, when the maximum deflection reaches or exceeds its thickness, membrane force will be induced. The large deflection effect in these cases is essentially represented by the membrane force effect. The membrane force induced by large deflection dissipates energy and stiffens the structure.

From the viewpoint of energy equilibrium, a theoretical procedure called Membrane Factor Method was proposed by the authors (see, e.g. [9, 10, 14]) in analyzing the dynamic plastic response of beams and plates with deflections in the range where both bending moments and membrane forces are of significance. In this paper, the Membrane Factor Method is generalized and elaborated and its applications to various cases of dynamically loaded beams and plates are surveyed. General characteristics of this method are demonstrated.

\section{Membrane Factor Method}

Consider a beam with end axial constraints or a plate secured along its contour, which is assumed to be made of a rigid, perfectly plastic material. Let $M_{0}$ denote its fully plastic bending moment. When the deflection reaches the order of its thickness, the influence of large deflection is essentially represented by the membrane (axial) force effect. From the viewpoint of energy dissipation, taking account of the contribution of the membrane force induced by large deflection is equivalent to regarding the beam or plate as one having a plastic bending moment that varies with the deflection. Hence, we may introduce a factor $f_{n}$, which represents the effect of membrane force and depends on the deflection of the beam or plate, to modify the equations of motion based on small deflection assumption by a change $M_{0} \rightarrow M_{0} f_{n}$, which implies that the energy dissipation at a hinge in the beam or plate is modified (enlarged); in other words, the 
bending moment at a plastic hinge is now assumed to be $M_{0} f_{n}$ rather than $M_{0}$. Accordingly, the equations of motion for large deflection are straightforwardly formulated.

From energy equilibrium principle, the modifying factor $f_{n}$ that accounts for the effect of the membrane force induced by large deflection can be defined as

$$
f_{n}=J_{m n} / J_{m},
$$

where $J_{m n}$ is the plastic energy dissipation rate due to both bending and membrane stretching using interactive yield condition between bending moment and membrane force, $J_{m}$ is the dissipation rate merely due to bending from small deflection theory.

\section{Case studies of beams}

\subsection{Beams under impulsive loading}

Consider a fully-clamped or a simply supported beam of length $2 L$ and mass per unit length $m$, subjected to an impulsive pressure resulting in a uniformly distributed velocity $V_{0}$ (as shown in Fig. 1). As is known, the deformation mechanism is constructed by plastic hinges and rigid segments, and the response history consists of two phases: the first (transient) phase, which is characterized by travelling plastic hinges, and the succeeding (modal) phase, with all plastic hinges stationary. The so-called plastic hinge here means a generalized one under the interaction between bending moment $M$ and axial force $N$ (Fig. 2).
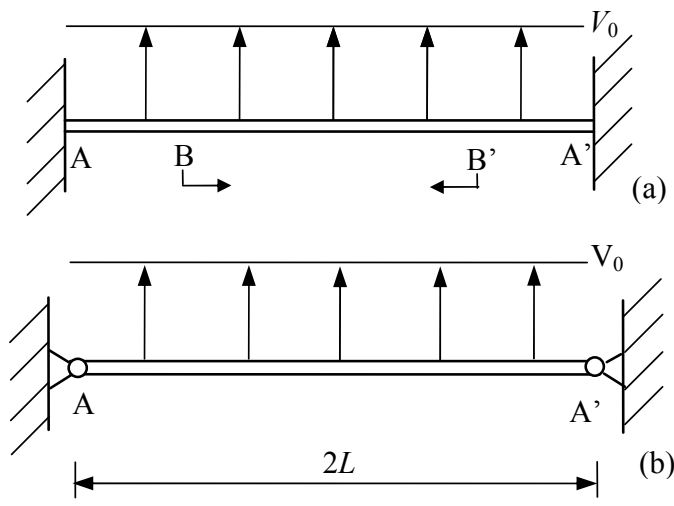

Figure 1: Beams under impulsive loading: (a) fully-clamped; (b) simply supported with end axial constraints.

Using the Membrane Factor Method, the equations of motion for large deflection of fully-clamped beam can be easily found as [10]

$$
\omega \xi L=V_{0},
$$




$$
\int_{0}^{\xi L} \omega x^{2} m d x+\int_{\xi L}^{L} V_{0} x m d x-\int_{0}^{L} V_{0} x m d x=-2 M_{0} \int_{0}^{t} f_{n} d t
$$

in the first phase, and

$$
m L^{3} \dot{\omega} / 3=-2 M_{0} f_{n}
$$

in the second phase. For equations of motion of the simply supported beam with end axial constraints, the only change is the replacement of $2 M_{0}$ by $M_{0}$ in eqns. (3) and (4). The governing equations (2)-(4) are close-formed, provided that the membrane factor $f_{n}$ is determined.

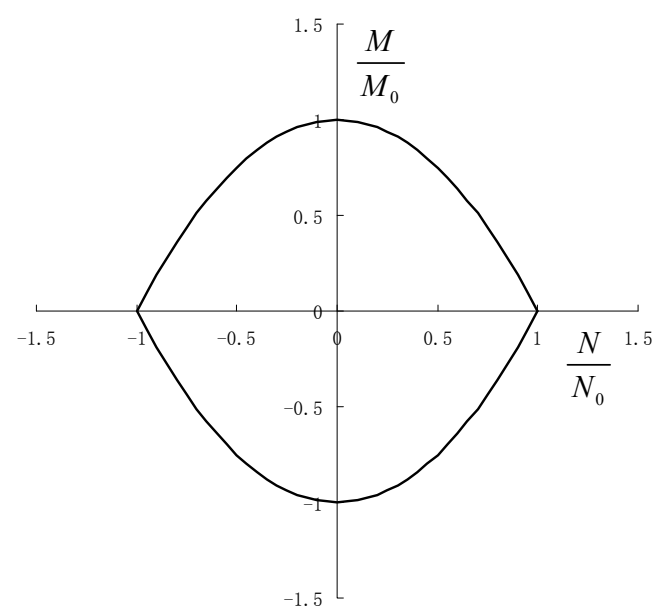

Figure 2: Interactive plastic yield condition.

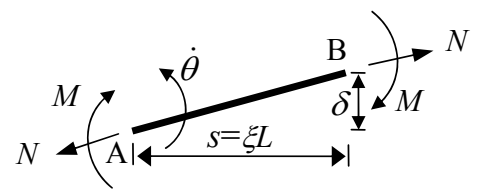

Figure 3: Forces on segment AB.

The key of formulation is to find the membrane factor $f_{n}$. For the fullyclamped beam, referring to segment $\mathrm{AB}$ in Fig. 3 (B is a travelling plastic hinge) and letting $s=\xi L$, the elongation rate of half the beam is

$$
\dot{\Delta}=\lim _{\Delta t \rightarrow 0} \frac{\sqrt{(s+\Delta s)^{2}+(\delta+\Delta \delta)^{2}-\left(\sqrt{s^{2}+\delta^{2}}+\Delta s\right)}}{\Delta t} .
$$

With deflection $\delta<<s$, it reduces to $\dot{\Delta} \approx \delta \dot{\theta}$. From the interactive plastic yield condition and associated normal flow rule $d M / d N=-\dot{\Delta} / 2 \dot{\theta}$, we find at generalized plastic hinges $\mathrm{A}$ and $\mathrm{B}$, 


$$
M / M_{0}=1-\eta^{2}, N / N_{0}=\eta
$$

when $\eta \equiv \delta / H \leq 1$. It follows that the plastic dissipation rate in half the beam due to both bending and axial stretching is

$$
J_{m n} / 2=2 M \dot{\theta}+N \dot{\Delta}=2 M_{0} \dot{\theta}\left(1+\eta^{2}\right) .
$$

On the other hand, bending-only analysis results in $M=M_{0}$ and $N=0$ at the plastic hinges, as a result the plastic dissipation rate in half the beam merely due to bending is

$$
J_{m} / 2=2 M_{0} \dot{\theta}
$$

Substituting eqns. (7) and (8) into (1) yields

$$
f_{n}=1+\eta^{2},
$$

for $\eta=\delta / H \leq 1$. When $\eta=\delta / H \geq 1, M=0$ and $N=N_{0}$, as a result, $f_{n}=2 \eta$. It is convenient to express the membrane factor in a complete form

$$
f_{n}=\left\{\begin{array}{l}
1+\eta^{2}, \quad \text { when } \eta \leq 1 \\
2 \eta, \quad \text { when } \eta \geq 1
\end{array}\right.
$$

Similarly, for the simply supported beam, noting that its two ends are natural hinges instead of plastic ones, it turns out that

$$
f_{n}=\left\{\begin{array}{l}
1+4 \eta^{2}, \quad \text { when } \eta \leq 1 / 2 \\
4 \eta, \quad \text { when } \eta \geq 1 / 2
\end{array}\right.
$$

From eqns. (10) and (11), it is seen that $f_{n}$ merely depends on the dimensionless central deflection $\eta=\delta / H$.

Solving eqns. (2)-(4) coupling with the complementary equation (10) yields the final dimensionless deflection $\eta_{f}=\delta_{f} / H$ as

$$
\eta_{f}+\frac{1}{3} \eta_{f}^{3}=\frac{1}{6} \lambda \text {, if } \lambda \leq 8 ; \quad \eta_{f}=\frac{1}{\sqrt{6}} \sqrt{\lambda-2} \text {, if } \lambda \geq 8
$$

For the fully-clamped beam, in which $\lambda=m L^{2} V_{0}^{2} / M_{0} H$ is a dimensionless impact parameter. Similarly, for the simply supported beam,

$$
\eta_{f}+\frac{4}{3} \eta_{f}^{3}=\frac{1}{3} \lambda, \text { if } \lambda \leq 2 ; \quad \eta_{f}=\frac{1}{\sqrt{6}} \sqrt{\lambda-\frac{1}{2}}, \text { if } \lambda \geq 2
$$

It is seen that by the Membrane Factor Method, the large deflection solution has a succinctly analytical form.

\subsection{Beam-on-foundation under impact}

$\mathrm{Yu}$ and Stronge [9] first tentatively introduced membrane factor to represent the large deflection effect in the dynamic response of a rigid-plastic beam-onfoundation from impact (Fig. 4). The membrane factor was found to have the same form of eqn. (10), where $\eta=w_{0} / H$ and $w_{0}$ is deflection at the impact point.

With the modifying membrane factor, equations of motion for large deflection analysis were derived as [9] 


$$
\left\{\begin{array}{l}
(G+2 \rho b+\rho \lambda) \dot{V}=-2 q_{0} f_{q}(b+\lambda) ; \\
\rho \lambda^{2} \dot{V}+2 \rho \lambda V \dot{\lambda}=12 M_{0} f_{n}-3 q f_{q} \lambda^{2} .
\end{array}\right.
$$

Eqns. (14) were solved by a Runge-Kutta integration procedure, so that the entire response history (including a transient phase) can be traced (see Fig. 5).
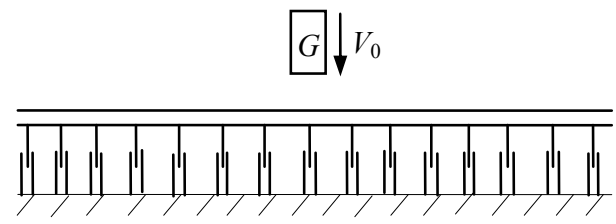

Figure 4: Impact against rigid-plastic beam on perfectly plastic foundation.

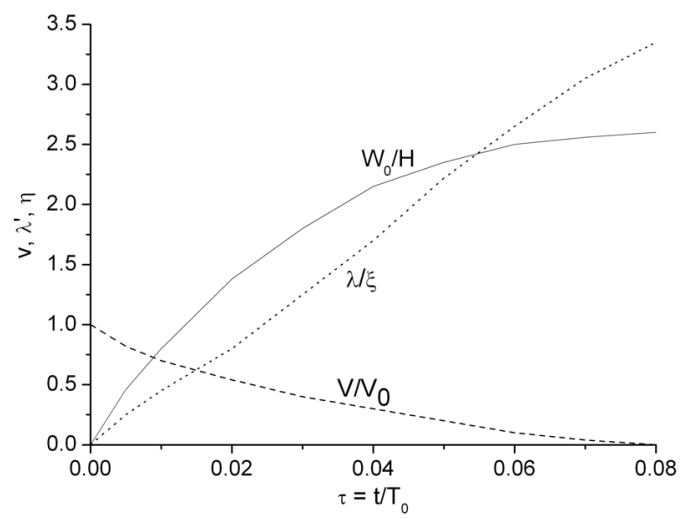

Figure 5: Response history of a beam-on-foundation after impact.

\subsection{Fully-camped beam under impact load}

A fully-clamped beam struck transversely at its mid-span by a mass was examined in [11], as illustrated in Fig. 6. After impact, the striker is supposed to

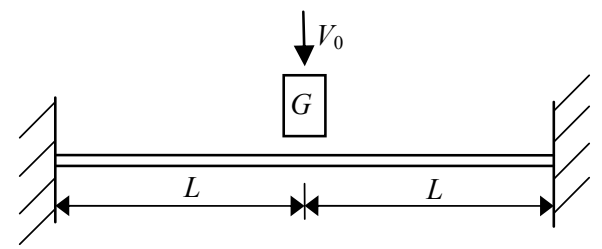

Figure 6: A fully-clamped beam subjected to impact load. 
adhere to the beam always. The membrane factor was found to have the same form of eqn. (10) and the equations of motion for large deflection are

$$
\left\{\begin{array}{l}
(G+m s) \ddot{Z}+m \dot{s} \dot{Z}=0 \\
m s^{2} \ddot{Z}+2 m s \dot{s} \dot{Z}=12 M_{0} f_{n}
\end{array}\right.
$$

for the first transient phase; and

$$
\int_{0}^{L} \frac{1}{2} m v_{1}^{2} d x+\frac{1}{2} \cdot \frac{1}{2} G V_{1}^{2}=\int_{\eta_{1}}^{\eta_{f}} 2 M_{0} f_{n}(\eta) d \eta
$$

for the succeeding modal phase. The final deflection of the beam is analytical:

$$
\begin{cases}\eta_{f}+\eta_{f}^{3} / 3=\Omega, & \text { if } \left.\Omega \leq 4 / 3 \text { (i.e. } \eta_{f} \leq 1\right) \\ \eta_{f}=\sqrt{\Omega-1 / 3}, & \text { if } \left.\Omega \geq 4 / 3 \text { (i.e. } \eta_{f} \geq 1\right)\end{cases}
$$

where $\Omega \equiv \frac{\beta \lambda}{12 \alpha}\left[\ln \left(1+\frac{1}{\beta}\right)+\frac{1}{2(\beta+1)}\right]$, and $\alpha \equiv \frac{H}{L}, \beta \equiv \frac{G}{m L}, \lambda \equiv \frac{G V_{0}^{2}}{M_{0}}$.

\section{Case studies of plates}

\subsection{Circular plates}

Consider a simply supported circular plate subjected to an impulse resulting in uniformly distributed velocity, as shown in Fig. 7. Two membrane factors corresponding respectively to the $r$ and $\theta$ direction were deducted as [12]

$$
f_{n r}=\left\{\begin{array}{l}
1+4 \eta^{2}, \quad \text { when } \eta \leq 1 / 2 ; \\
4 \eta, \quad \text { when } \eta \geq 1 / 2
\end{array} ; f_{n \theta}= \begin{cases}1+4 \eta^{2} / 3, & \text { when } \eta \leq 1 / 2 ; \\
2 \eta+1 / 6 \eta, & \text { when } \eta \geq 1 / 2 .\end{cases}\right.
$$

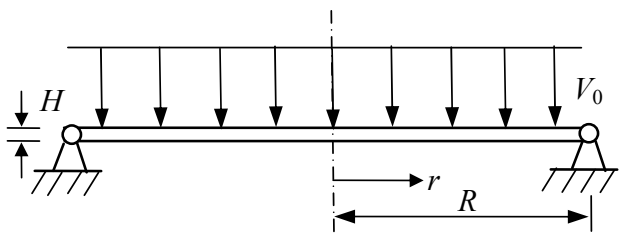

Figure 7: A simply supported circular plate subjected to impulsive loading.

So the equation of motion for large deflection was established as

$$
\left(r M_{r} f_{n r}\right)^{\prime}=M_{\theta} f_{n \theta}+\int_{0}^{r} \mu \ddot{w} r d r
$$

where ()$^{\prime}=\partial() / \partial r$. As a result, final deflection of the plate was found as 


$$
\begin{cases}\left(1+\frac{24}{\lambda} \tau_{1}+\frac{32}{3 \lambda} \tau_{1}^{3}\right)-\frac{24}{\lambda} \eta_{f}-\frac{32}{3 \lambda} \eta_{f}^{3}=0 & \left(\tau_{1} \leq \frac{1}{2}, \zeta \leq 0\right) \\ 1-\frac{22}{3 \lambda}+\frac{24}{\lambda} \tau_{1}+\frac{32}{3 \lambda} \tau_{1}^{3}-\frac{24}{\lambda}\left(\eta_{f}^{2}-\frac{1}{6} \ln \left(2 \eta_{f}\right)\right)=0 & \left(\tau_{1} \leq \frac{1}{2}, \zeta \geq 0\right) \\ 1-\frac{24}{\lambda}\left(\eta_{f}^{2}-\tau_{1}^{2}+\frac{1}{6}\left(\ln \eta_{f}-\ln \tau_{1}\right)\right)=0 & \left(\tau_{1} \geq \frac{1}{2}\right)\end{cases}
$$

where $\lambda=\mu V_{0}^{2} R^{2} / M_{0} H, \tau_{1}, \zeta$ are dimensionless parameters.

\subsection{Regular polygonal plates}

An $n$-side (e.g. $n=6$ ) regular polygonal plate with simply supported or fully clamped edges are subjected to an impulsive loading, its deformation pattern is illustrated in Fig. 8 with $n$ plastic hinge lines travelling inward until shrinking at the centre [13]. Refer to [3], the plastic dissipation rate due to the interaction between bending moment and membrane force along a plastic hinge line in a plate is $D_{m n}=(N w-M) \dot{\theta}_{i}$, while the plastic dissipation rate due to bending-only is $D_{m}=M_{0} \dot{\theta}_{i}$. Therefore, the membrane factor was found as

$$
f_{n}=\left\{\begin{array}{l}
1+4(1+2 \xi) \eta^{2} / 3, \quad \text { when } \eta \leq 1 / 2 \\
2 \eta+1 / 6 \eta+\xi(2 \eta-1 / 6 \eta), \quad \text { when } \eta \geq 1 / 2
\end{array}\right.
$$

for simply supported edges, and

$$
f_{n}=\left\{\begin{array}{l}
1+(1-\xi) \eta / 2+\xi \eta^{2} 3 \xi \eta^{2} / 2, \quad \text { when } \eta \leq 1 \\
1 / 2+(1+\xi) \eta, \quad \text { when } \eta \geq 1
\end{array}\right.
$$

for fully-camped edges, in which $\xi$ is a parameter indicating the position of the inward travelling plastic hinge lines.

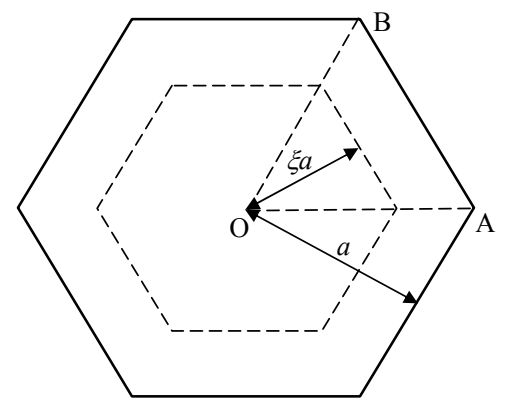

Figure 8: Deformation pattern of $n$-side (e.g. $n=6$ ) regular polygonal plate under impulsive loading.

It turns out that the final central deflection of a regular polygonal plate with simply supported edges is 


$$
\begin{cases}4\left(\eta_{f}^{3}-\eta_{1}^{3}\right) / 9+\left(\eta_{f}-\eta_{f}\right)-\lambda / 24=0 & \left(\eta_{1} \leq 1 / 2, R_{1} \leq 0\right) \\ \left(\eta_{f}^{2}-1 / 4\right)+\ln \left(2 \eta_{f}\right) / 6-\lambda R_{1} / 24=0 & \left(\eta_{1} \leq 1 / 2, R_{1} \geq 0\right) \\ \left(\eta_{f}^{2}-\eta_{1}^{2}\right)+\ln \left(\eta_{f} / \eta_{1}\right) / 6-\lambda / 24=0 & \left(\eta_{1} \geq 1 / 2\right)\end{cases}
$$

where $R_{1}=1-\frac{24}{\lambda}\left[\left(\frac{1}{2}-\eta_{1}\right)+\frac{4}{9}\left(\frac{1}{8}-\eta_{1}^{3}\right)\right]$, and $\lambda=\frac{\mu V_{0}^{2} a^{2}}{M_{0} H}$. For the case of fullycamped edges,

$$
\begin{cases}\left(\eta_{f}^{2}-\eta_{1}^{2}\right)+4\left(\eta_{f}-\eta_{f}\right)-\lambda / 12=0 & \left(\eta_{1} \leq 1, R_{2} \leq 0\right) \\ \eta_{f}^{2}+\eta_{f}-\left(2+\lambda R_{2} / 24\right)=0 & \left(\eta_{1} \leq 1, R_{2} \geq 0\right) \\ \left(\eta_{f}^{2}-\eta_{1}^{2}\right)+\left(\eta_{f}-\eta_{1}\right)-\lambda / 24=0 & \left(\eta_{1} \geq 1\right)\end{cases}
$$

where $R_{2}=1-(48 / \lambda)\left[\left(1-\eta_{1}\right)+\left(1-\eta_{1}^{2}\right) / 4\right]$.

\subsection{Rectangular plates}

Rectangular plates are of more practical value to engineers. For a rectangular plate secured along its entire contour subjected to a uniformly distributed load of intensity $P(t)$, the deformation mechanisms are shown in Fig. 9, where dashed are plastic hinge lines. Two membrane factors corresponding to the horizontal and vertical direction were derived as [14]

$$
\begin{aligned}
& f_{n 1}=\left\{\begin{array}{l}
{\left[2+(3-4 \delta) \eta^{2}\right] / 2, \quad \text { when } \eta \leq 1 ;} \\
{[1+4(1-\delta) \eta] / 2, \quad \text { when } \eta \geq 1 ;}
\end{array}\right. \\
& f_{n 2}=\left\{\begin{array}{l}
{\left[2+(3-4 \xi) \eta^{2}\right] / 2, \quad \text { when } \eta \leq 1 ;} \\
{[1+4(1-\xi) \eta] / 2, \quad \text { when } \eta \geq 1}
\end{array}\right.
\end{aligned}
$$

for fully-camped edges, in which $\xi$ and $\delta$ denote the position of travelling plastic hinge lines.

Thus, equations of motion for large deflection are

$$
\left\{\begin{array}{l}
\ddot{\phi}_{1} \xi^{3}(2-3 \delta)=p(\tau)(3-4 \delta) \xi^{2}-6 \alpha f_{n 1} ; \\
\ddot{\phi}_{2} \delta^{3}(2-3 \xi)=p(\tau)(3-4 \xi) \delta^{2}-6 \gamma^{2} \beta f_{n 2} ; \\
d\left(\dot{\phi}_{1} \xi\right) / d \tau=p(\tau) ; \\
\dot{\phi}_{1} \xi=\dot{\phi}_{2} \delta .
\end{array}\right.
$$

For the special case of impulsive loading, eqns. (27) were reduced and then solved by integrating with time to trace the entire large deflection dynamic plastic response (including a transient phase) [14]. The role of plastic energy dissipation by membrane forces in the dynamic plastic response of rectangular plates was further examined by the authors in [15]. 

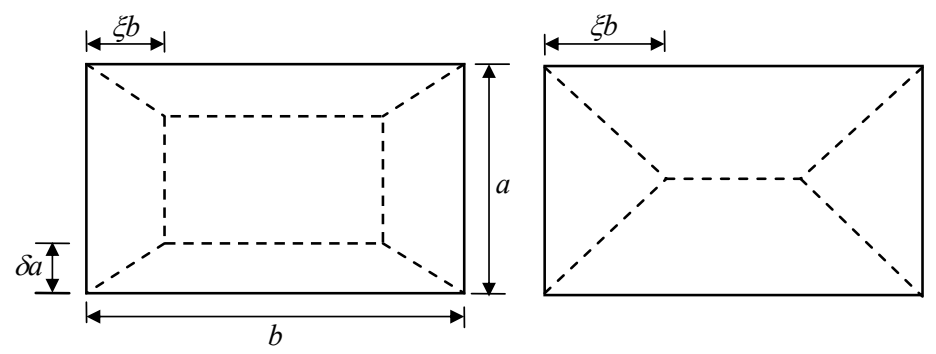

Figure 9: Deformation mechanisms of a rectangular plate.

\section{Discussion and comparison of results}

The above case studies show that the Membrane Factor Method (MFM) provides an effective way to deal with the large deflection plastic response of structures subjected to intense dynamic loads. The merits of this approach are obvious: (i) it can easily trace the entire response history including a first transient phase and succeeding modal phase; (ii) the membrane factors can usually be expressed as analytical functions of dimensionless deflection of beams or plates, for plates parameters describing the position of plastic hinge lines may be involved; (iii) the established governing equations or solutions on the final deflection are usually in favourably succinct form.

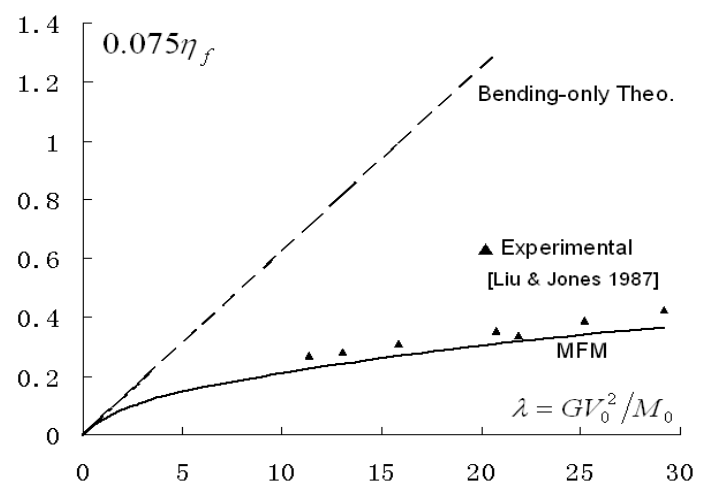

Figure 10: Comparison of results of beam under impact load.

Figure 10 presents a comparison of theoretical predictions on final deflection of fully-clamped beam subjected to impact load with experiment. It is seen that while the bending-only solution deviates far away from the experimental records once the deflection reaches the order of its thickness, the large deflection solution by MFM, although in very succinct form (eqn. (17)), agrees well with the experimental results when the deflection is several times of beam thickness.

A comparison of the results for simply supported circular plates subjected to impulsive loading is shown in Fig. 11. It is seen that when the final deflection 


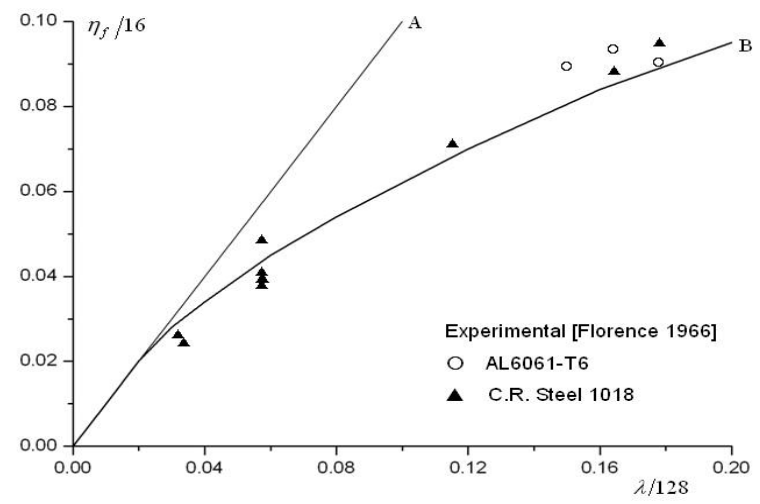

Figure 11: Final deflection of circular plates subjected to impulsive loading: A: bending-only theory [2]; B: solution by the MFM.

reaches the order of plate thickness, the theoretical predictions by MFM agrees well with experimental results by Florence [7], whereas the bending-only theory incurs obvious discrepancy.

Figure 12 gives a comparison of the theoretical predictions on final deflection of impulsively loaded rectangular plates with corresponding experimental results. A, B are upper bounds based on small deflection analysis by Jones et al. [8]; C, $\mathrm{C}^{\prime}$ are upper and lower bounds based on a small deflection analysis by Komarov

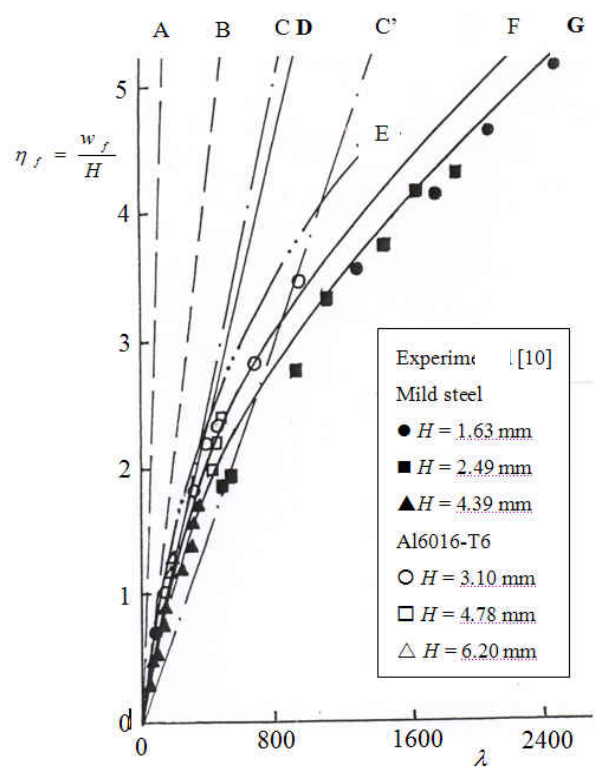

Figure 12: Comparison of theoretical predictions on final deflection of fullyclamped rectangular plates with experimental results. 
and Nemirovskii [16]; $\mathrm{D}$ is bending-only theory solution (i.e. $f_{n} \equiv 1$ ); $\mathrm{E}$ is approximate solution of finite deflection based on modal deformation profile [3]; $\mathrm{F}$ is large deflection solution by the Membrane Factor Method; $G$ is solution in [15]. Experimental results are taken from [8]. It once again verifies that the Membrane Factor Method can give good theoretical predictions for deflections up to more than 5 times of the plate thickness.

\section{Conclusion}

From the viewpoint of energy equilibrium, a theoretical procedure called Membrane Factor Method was proposed by the authors in analyzing the dynamic plastic response of beams and plates with deflections in the range where both bending moments and membrane forces are of significance. Case studies show that the predictions by this method on the final deflections of beams and plates agree well with corresponding experimental results for deflections up to 5-10 times of thickness. This procedure greatly improves the estimates from the bending-only theory and provides feasible complete response (including the transient phase) analyses, whose predictions are much more reliable than modal approximations. Furthermore, this method is applicable to more complicated structures [17].

\section{References}

[1] Jones, N., Structural Impact, Cambridge University Press: New York, Paperback Ed, 1997, 2nd Ed, 2012.

[2] Wang, A.J., The permanent deflection of a plastic plate under blast loading. J. Appl. Mech., 22, pp. 375-376, 1955.

[3] Jones, N., A theoretical study of the dynamic plastic behaviour of beams and plates with finite deflections. Int. J. Solids Struct., 7, pp. 1007-1029, 1971.

[4] Jones, N., Impact loading of ductile rectangular plates. Thin-Walled Struct., 50, pp. 68-75, 2012.

[5] Baker, W.E., Approximate techniques for plastic deformation of structures under impulsive loading. Shock Vib. Dig., 1975, 7, pp. 107-117, 1975.

[6] Jiu, J.H. \& Jones, N., Experimental investigation of clamped beams struck transversely by a mass. Int. J. Impact Engng, 6, pp. 303-335, 1987.

[7] Florence, A.L., Circular plates under a uniformly distributed impulse. Int, J. Solids Struct., 2, pp. 37-47, 1966.

[8] Jones, N., Uran, T., \& Tekin, S.A., The dynamic plastic behaviour of fully clamped rectangular plates. Int. J. Solids Struct., 6, pp. 1499-1512, 1970.

[9] Yu, T.X. \& Stronge W.J., Large deflections of a rigid-plastic beam-onfoundation from impact. Int. J. Impact Engng., 9, pp. 115-126, 1990.

[10] Chen, F.L. \& Yu, T.X., Analysis of large deflection dynamic response of rigid plastic beams. ASCE Int. J. Eng. Mech., 119, pp. 1293-1301, 1993. 
[11] Chen, F.L. \& Yu, T.X., Large plastic deflection of fully clamped beams subjected to impact. Mater. Res. Innov., 15, pp. 195-197, 2011.

[12] Yu, T.X. \& Chen, F.L., Analysis of the large deflection dynamic response of simply-supported circular plates by the membrane factor method. Acta Mechanica Sinica (English Edition), 6, pp. 333-342, 1990.

[13] Chen, F.L. \& Yu, T.X., Dynamic plastic response of regular polygonal plates. Explosion and Shock Waves, 11, pp. 106-116, 1991.

[14] Yu, T.X. \& Chen F.L., The large deflection dynamic plastic response of rectangular plates. Int. J. Impact Engng., 12, pp. 603-616, 1992.

[15] Chen, F.L. \& Yu, T.X., Dynamic plastic response of rectangular plates with plastic energy dissipation by membrane forces. Explosion and Shock Waves, 25, pp. 200-206, 2005.

[16] Komarov, K.L. \& Nemirovskii, Y.V., Dynamic behaviour of rigid-plastic rectangular plates. UDC 624.072.22, 21(7): pp. 69-76, 1985.

[17] Xiang, X.M. \& Lu, G.X., Dynamic deflection of a beam on metal foam. Key Eng. Mater., 535-536, pp. 481-484, 2013. 